\title{
Editorial
}

\section{Multisite stimulation for correction of cardiac asynchrony}

Cardiac electrical stimulation as primary or adjunctive treatment of congestive heart failure is entering its second decade of existence. Initial trials of conventional DDD pacing $^{1-5}$ were followed by bifocal right ventricular stimulation. ${ }^{6}$ The concept of multisite stimulation for haemodynamic support was introduced in $1994 . .^{78}$ Various studies have already reported the benefits of this method, ${ }^{9}{ }^{10}$ or are in progress. ${ }^{11}$

The transition from conventional DDD pacing to multisite stimulation was not fortuitous. Successive "advances" in the design of cardiac pacing systems have aimed at correcting anomalies in the sequence of cardiac chamber activation, as well as in the synchronisation of the various phases of myocardial contraction and relaxation. The phenomenon of asynchrony is a consequence of progressive, global or focal degradation of the myocardium. One can easily visualise interstitial fibrosis gradually replacing areas of normal myocardium, and causing heterogeneous propagation of cardiac electrical activity. Such heterogeneity combines, to various degrees, three consecutive atrioventricular, interventricular, and intraventricular asynchrony levels. Among various analytical and modelling methods, Doppler echocardiography is preferred for its ease of application in day to day practice. ${ }^{12}$

\section{Atrioventricular asynchrony}

The oldest concept, that of desynchronised atrioventricular sequential activation, applies only to sinus rhythm. It is the product of a mismatch between end of atrial systole and onset of ventricular systole, sometimes facilitated by a disorder of atrioventricular conduction or QRS prolongation. It may be simply described as an abbreviated ventricular filling time with respect to the complete cardiac cycle, and, on occasion, by early passive ventricular filling flow superimposed on atrial systole dependent flow. Dual chamber pacing, by linking ventricular to atrial activation, normalises flow patterns of ventricular filling, provided atrial contraction is mechanically effective (fig 1). This principle

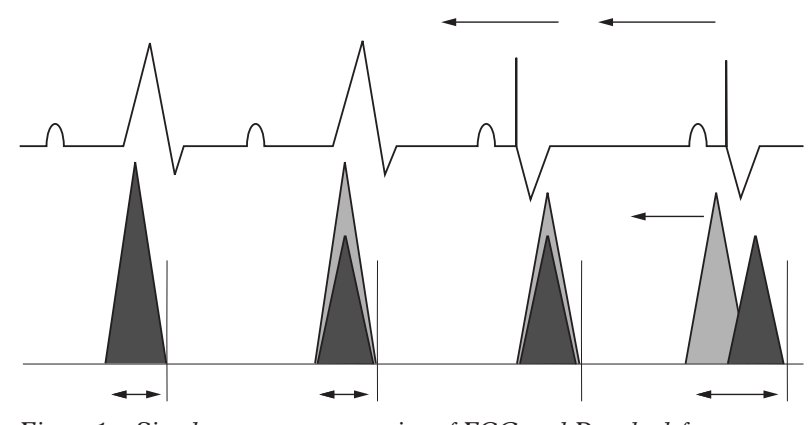

Figure 1 Simultaneous representation of ECG and Doppler left ventricular diastolic filling flows. The first two complexes show spontaneous rhythm. The left ventricular contraction delay caused by a prolonged PR interval and, perhaps, prolonged $Q R S$ duration, is responsible for a delay in ventricular systole with respect to atrial systole. This delay postpones the $E$ wave, now superimposed upon the $A$ wave. The pre-excitation caused by shortening of the $A V$ delay compared to the native PR interval has no effect in the first ventricular paced cycle. However, with the second paced cycle, the $E$ wave location has changes with respect to the $A$ wave, and the ventricular filling time has lengthened. The $A$ wave position remains fixed within the cardiac cycle. guides the optimisation of the atrioventricular delay under Doppler echocardiographic guidance in dual chamber pacing. The end point consists of shortening the atrioventricular delay to a minimum without interrupting the end of the A wave by premature mitral valve closure (fig 2 ).

\section{The concept of interventricular asynchrony}

Atrioventricular resynchronisation alone has not been predictably effective in improving haemodynamics except in some patients with prolonged PR interval. ${ }^{2}$ Candidates for the implantation of a cardiac stimulator often present with intraventricular conduction disorders expressed as a widened QRS, an anomaly which may also be caused by the pacemaker itself. In the hope of minimising atrioventricular asynchrony, a delay is unfortunately imposed between right and left ventricular activation by stimulating the ventricle from the peripheral Purkinje network instead of preserving the normal sequence via the His bundle. This activation/contraction delay causes a delay in subsequent chamber relaxation, disturbing its filling (fig 3 ). As a result, the right-to-left electromechanical delay caused by typical pacing from the right ventricular apex may disrupt the synchrony of right and left ventricular systole. This spontaneous or iatrogenic asynchrony can be easily quantified by measuring the delay between the onset of ventricular electrical activation and the onsets of aortic and pulmonic ejection, respectively. The difference between these two intervals represents the interventricular mechanical delay (fig 4), and a shortening in both the pre-ejection delays and the interventricular delay appears to predict a benefit from multisite stimulation (fig 5). ${ }^{12}$

\section{The concept of intraventricular systolo-diastolic asynchrony}

Finally, heterogeneity of intraventricular myocardial contraction may be so pronounced as to result, within the same ventricle, in the coincidence of territories in systolic phase,
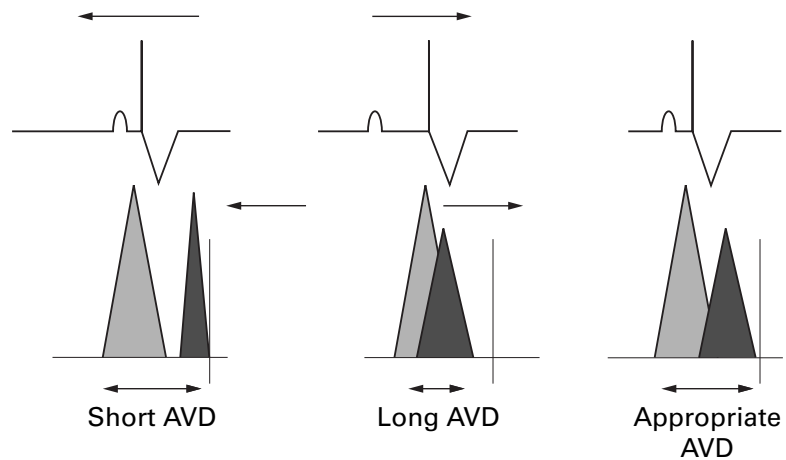

Figure 2 Left: the atrioventricular delay (AVD) is too short. The E wave is early, which tends to lengthen the ventricular filling duration; however, the $A$ wave is prematurely interrupted by mitral valve closure caused by ventricular systole. Centre: the atrioventricular delay is too long. $E$ and $A$ waves are superimposed, and filling time is abbreviated. The end of the $A$ wave is preserved, although a long interval to mitral valve closure may facilitate diastolic mitral regurgitation. Right: the atrioventricular delay is appropriate. It is the shortest atrioventricular interval which is not encroaching upon the end of the $A$ wave. 


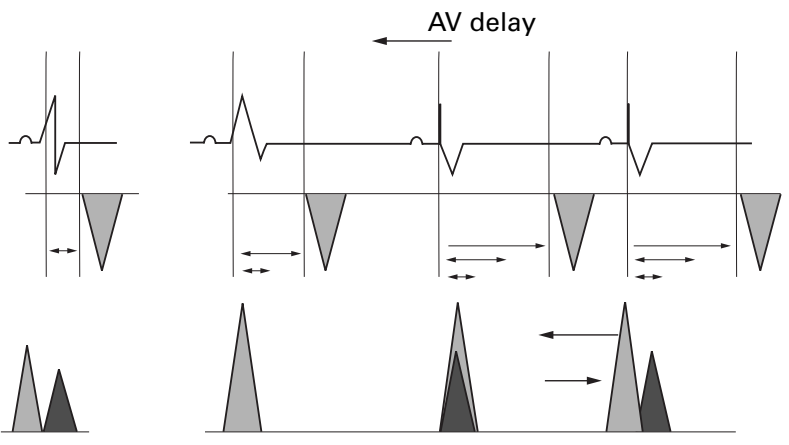

Figure 3 The first cycle illustrates the measurement between QRS onset (at the pacing spike) and onset of aortic ejection. With left bundle branch block (second spontaneous QRS), the pre-ejection interval is prolonged. Initiation of dual chamber pacing, which shortens the atrioventricular delay, intends to optimise ventricular filling and improve overall haemodynamics. However, the pacing system itself causes a right-to-left activation delay which further prolongs the pre-ejection interval. While trying to optimise diastole, systole was lengthened.

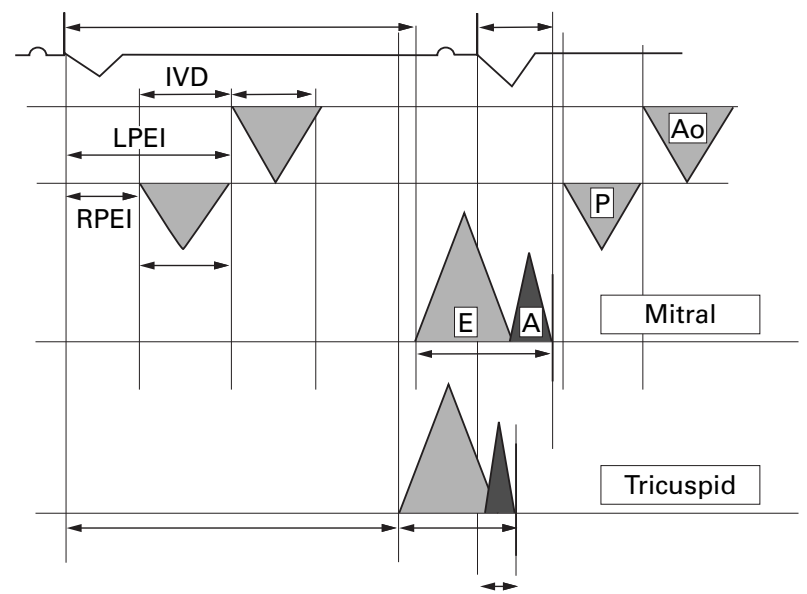

Figure 4 The mechanical interventricular delay $(I V D)=L P E I-R P E I$. It can routinely be measured, regardless of the ambient spontaneous or paced rhythm. LPEI, left pre-ejection interval; RPEI, right pre-ejection interval.

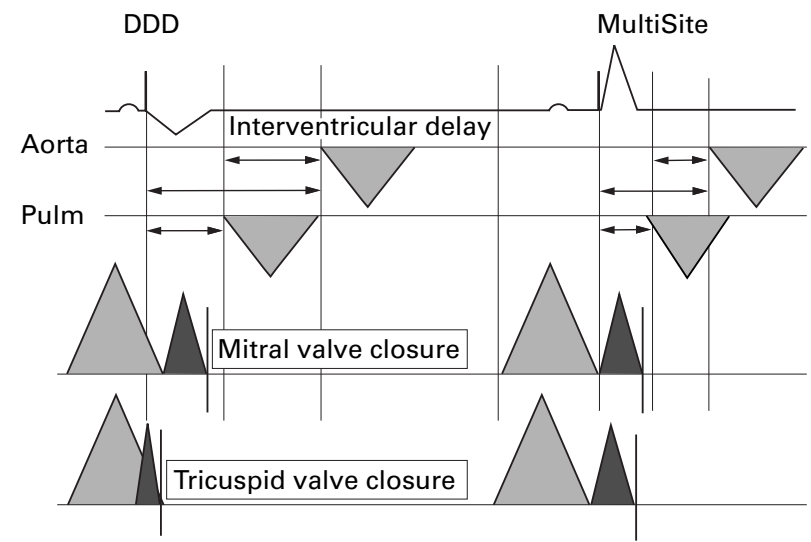

Figure 5 With biventricular stimulation, the left ventricle is activated earlier and, if the implant was "successful", the left pre-ejection interval is shorter compared to the interval measured during conventional right ventricular pacing. The atrioventricular delay no longer needs to be set at very short values in order to lengthen the ventricular filling time. Furthermore, total duration of systole is shortened instead of lengthened.

while others are already relaxing. ${ }^{13}$ A marker of such heterogeneity is the QRS width and, sometimes, its polyphasic morphology. Intraventricular asynchrony is unlikely in presence of spontaneous QRS complexes $<120 \mathrm{~ms}$, and paced QRS complexes < $180 \mathrm{~ms}$ in duration, and may be greatly accentuated by stimulation confined to the right heart chambers. It is often present in ischaemic cardiomyopathy, where the propagation of

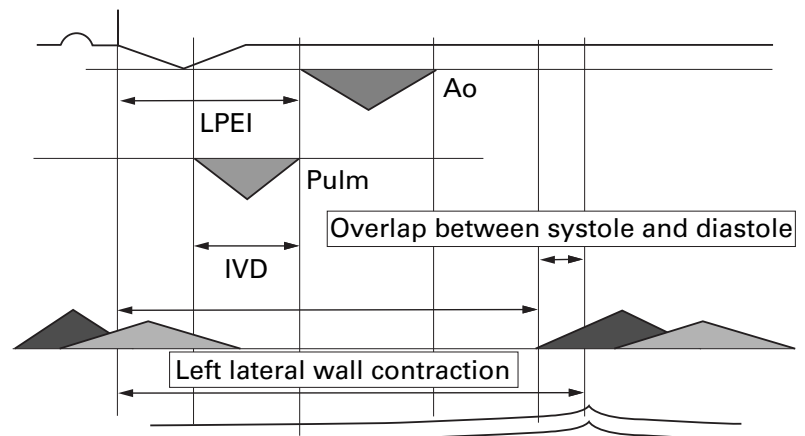

Figure 6 Four chamber apical view of the long axis shortening of the left ventricle. Time-movement evaluation of the contraction of the left lateral wall close to the mitral valve annulus is performed. The longest delay reflecting to the end of lateral wall systolic thickening is measured with respect to the QRS onset, and is compared with the obligatory delay to the onset of the following $E$ wave. In the case of intraventricular asynchrony, intraventricular contraction of the lateral wall may end after the beginning of ventricular filling, a source of a dual anomaly: (1) part of ventricular contraction is not utilised toward ejection; and (2) filling is impeded by persistent shortening along the long axis. In such patients, measurement of isovolumic relaxation duration is non-contributory since it does not reliably reflect segmental heterogeneity.

ventricular activation wavefronts may be disrupted by the encounter of scarred or ischemic tissue. This abnormal process is characterised by the presence of myocardial segments contracting as ventricular filling has already begun. This, on time-movement echocardiographic examination (fig 6), appears as segmental wall thickening occurring after the onset of the next early diastolic filling phase. Simultaneous stimulation of left and right cardiac chambers is, currently, not capable of predictably correcting this abnormality. However, its successful elimination often decreases the severity of mitral regurgitation, an important contribution to clinical improvement in some patients.

\section{Patient selection}

Multisite stimulation for congestive heart failure is a recent therapeutic method, which will, hopefully, be validated in the year 2000. It should be reserved for patients whose abnormal ventricular function may be corrected or improved by palliating conduction disorders, in the hope of changing the sequence of mechanical events, enhancing contractile efficiency, and, in some cases, lessening the severity of ventriculo-atrial regurgitation ${ }^{9}$ by changing systolic left ventricular geometry. Other mechanisms have been proposed, which remain speculative. Although the cardiac mechanics related to multisite stimulation are only partially understood, echocardiography has emerged as a non-invasive and easily reproducible test of choice in the selection of candidates for this new treatment. The absence of preimplant visible asynchrony probably predicts little improvement by multisite stimulation, but this point needs confirmation. Conversely determination of the nature of the asynchrony should facilitate the choice of an optimal synchroniser configuration. Modification of the electromechanical intervals in the postoperative course should also provide useful information for assessing the success or the failure of cardiac resynchronisation.

S CAZEAU

D GRAS

A LAZARUS

P RITTER

J MUGICA

InParys Clinical Research Associates, Saint-Cloud, Clinique Georges Bizet, Institut facques Cartier, Paris, France Sergecaz@aol.com 
1 Hochleitner $\mathrm{M}$, Hörtnagl $\mathrm{H}, \mathrm{NgCK}$, et al. Usefulness of physiologic dual-chamber pacing in drug-resistant idiopathic dilated cardiomyopathy. Am 7 Cardiol 1990;66:198-202.

2 Nishimura RA, Hayes DL, Holmes DR, et al. Mechanism of hemodynamic improvement by dual-chamber pacing for severe left ventricular dysfunction: an acute Doppler and catheterization hemodynamic study. $\mathcal{F}$ Am Coll Cardiol 1995;25:281-8.

3 Brecker SJ, Xiao HB, Sparrow J. Effects of dual-chamber pacing with short atrioventricular delay in dilated cardiomyopathy. Lancet 1992;340:130812 .

4 Linde C, Gadler F, Edner M, et al. Results of atrioventricular synchronous pacing with optimized delay in patients with severe congestive heart failure. Am F Cardiol 1995;75:919-23.

5 Gold MR, Feliciano Z, Gottlieb SS, et al. Dual-chamber pacing with a short atrioventricular delay in congestive heart failure: a randomized study. $\mathcal{F} \mathrm{Am}$ Coll Cardiol 1995;26:967-73.

6 Buckingham TA, Candidas R, Fromer M, et al. Acute hemodynamic effects of atrioventricular pacing at differing sites in the right ventricle individually of atrioventricular pacing at differing sites in the right ventricle individuall
and simultaneously [abstract]. Pacing Clin Electrophysiol 1995;18:1772.

7 Cazeau S, Ritter P, Bakdach S, et al. Four chamber pacing in dilated cardiomyopathy. Pacing Clin Electrophysiol 1994;17:1974-9.
8 Cazeau S, Ritter P, Lazarus A, et al. Multisite pacing for end-stage heart failure. PACE 1996;19:1748-57.

9 Leclercq C, Cazeau S, Le Breton H, et al. Acute hemodynamic effects of biventricular DDD pacing in patients with end-stage heart failure. $f \mathrm{Am}$ Coll Cardiol 1998;32:1825-31.

10 Auricchio A, Stellbrink C, Block M, et alKlein $\mathrm{H}$ for the Pacing Therapies for Congestive Heart Failure Study Group; Kramer A, Ding J, Salo R, et al, for the Guidant Congestive Heart Failure Research Group. Effect of pacing chamber and atrioventricular delay on acute systolic function of paced chamber and atrioventricular delay ocute systolic function of pace

11 Daubert C, Linde C, Cazeau S, et al on behalf the MUSTIC Study Group. Protocol design and current status of the European controlled multicenter study on multisite biventricular pacing in severe heart failure : the MUSTIC study [abstract]. PACE 1999;22:A116.

12 Cazeau S, Lazarus A, Ritter P, et al. Biventricular pacing decreases interventricular but not intraventricular asynchrony in multisite - paced patients for congestive heart failure [abstract]. PACE 1998;21:792.

13 Cazeau S, Lazarus A, Ritter P, et al. Overlap between systole and diastole in congestive heart failure patients candidates for multisite biventricular pacing [abstract]. PACE 1998;21:975.

\section{IMAGES IN CARDIOLOGY}

\section{Three dimensional reconstruction of femoral pseudoaneurysm using contrast enhanced axial CT angiography}

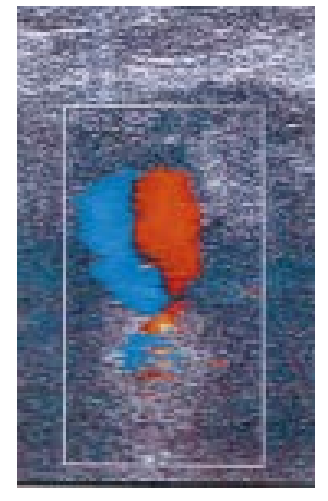

A 45 year old man was admitted for an electrophysiological procedure using a retrograde aortic approach via an 8 French right femoral artery sheath. The patient received low molecule weight heparin after the procedure. A large pulsatile mass with a diffuse haematoma and bruit developed in the right groin two days after the procedure. Duplex ultrasound scanning (ATL HDI 3000, 4-7 MHz probe) demonstrated a large cavity $(10 \times 18 \mathrm{~cm})$ with arterial turbulent flow (left). However, the origin of the false aneurysm from the artery could not be clearly identified. Contrast enhanced axial computed tomographic (CT) angiography with three dim-

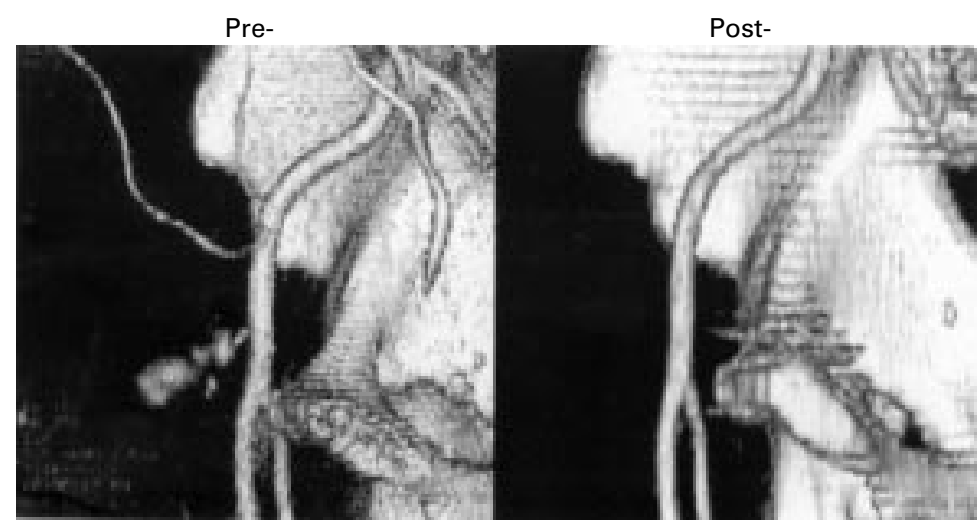

ensional reconstruction (Lightspeed CT scanner and Advantage Windows, Volume Rendering, General Electric, USA) was performed and confirmed a multiple saccular false aneurysm arising from the common femoral artery (below, left panel). The neck of the false aneurysm was localised by the CT angiogram and manual compression was applied at that site. After 20 minutes of compression, the pulsatility and the bruit from the false aneurysm ceased. A repeat CT angiogram confirmed the obliteration of the false aneurysm (below, right panel). The patient was discharged from the hospital the following day and only a small resolving haematoma was detected during follow up.

As this case illustrates, the presence of a multiple saccular false aneurysm may lead to difficulties in identifying its origin by duplex ultrasound scanning. CT angiogram is useful in such cases for diagnosing and guiding treatment of the iatrogenic pseudoaneurysm after femoral artery catheterisation.

HUNG-FAT TSE YUI-MING LAM MICHAEL YIU 